\title{
L'APPORT DES MONNAIES SOCIALES À LA MICROFINANCE : LE CAS DES BANQUES COMMUNAUTAIRES DE DÉVELOPPEMENT BRÉSILIENNES
}

\author{
Tristan DISSAUX* \\ CAMIlle MEYER $^{* *}$
}

$\mathrm{M}$ algré son insertion croissante au marché international des capitaux, le Brésil reste un pays marqué par une très forte exclusion bancaire et financière : $32 \%$ des Brésiliens adultes ne possèdent pas de compte bancaire ${ }^{1}$. Cette exclusion se décline selon de grandes disparités régionales, puisque les régions les plus développées (Sud, Sudeste et Centre-Ouest) affichent un taux de bancarisation de près de $70 \%$, alors que près d'un habitant sur deux ne possède pas de compte dans les régions Nordeste et Nord (Schiavinatto et de Holanda Schmidt, 2011). Du côté du crédit, seulement $12 \%$ de la population adulte est emprunteuse auprès d'une institution formelle. Cette problématique de l'exclusion financière a conduit la Banque centrale du Brésil (BCB) à considérer l'inclusion financière comme un enjeu crucial, sa réalisation pouvant être vecteur de développement économique et social. La microfinance doit participer à cette inclusion financière et le microcrédit en est encore la principale modalité.

Selon la BCB (2015), en 2014, le portefeuille national de microcrédits a atteint une valeur de 6,2 milliards de réaux brésiliens, soit

\footnotetext{
* Doctorant, laboratoire Triangle (UMR CNRS 5206), université Lyon 2. Contact : tristan.dissaux@ univ-lyon2.fr.

** Doctorant, université libre de Bruxelles, Solvay Brussels School, Centre Émile Bernheim, CERMi. Contact : cmeyer@ulb.ac.be. Recherche réalisée dans le cadre du Pôle d'attraction inter-universitaire "If Not for Profit, for What and How? » financé par la Politique scientifique fédérale belge (BELSPO).
} 
l'équivalent de 1,5 Md€, pour un total de 3,3 millions d'opérations. Ce montant représente une hausse de $16,6 \%$ par rapport à 2013 , soit une augmentation supérieure à celle du crédit total du système financier national. Les services de microcrédit sont fournis principalement par des banques publiques régionales (tels que CrediAmigo - deuxième programme de microcrédit en Amérique latine - offert par la Banque du Nordeste du Brésil), des sociétés privées de crédit aux microentrepreneurs, des coopératives de crédit, des organisations non gouvernementales $(\mathrm{ONG})$ ou des organisations locales sans but lucratif (Soares et de Melo Sobrinho, 2008).

Cet article s'intéresse plus particulièrement au modèle mis en œuvre par les banques communautaires de développement. L'une des principales innovations de ces organisations de la société civile est de coupler le microcrédit à l'émission d'une monnaie sociale, dont l'usage - en complément du réal brésilien - est circonscrit au territoire local. Nous verrons ce qu'apportent ces monnaies et dans quelle mesure elles viennent répondre à certaines des limites du microcrédit, à travers l'étude de cas de la Banque Palmas, fondatrice de ce modèle de finance solidaire.

\section{LA MICROFINANCE : LES LIMITES D'UN MODÈLE}

Malgré l'enthousiasme qui a accompagné le développement du microcrédit durant les trente dernières années, son impact sur la réduction de la pauvreté reste néanmoins une question toujours en suspens (Doligez et al., 2016). Ce problème de l'évaluation dure depuis les débuts du microcrédit et est régulièrement renouvelé à l'occasion de nouvelles études (Guérin, 2015).

On peut d'abord remarquer que la possibilité de tirer des conclusions claires quant aux bénéfices de la microfinance en général et du microcrédit en particulier apparaît au minimum incertaine. Sur la base de revues systématiques de la littérature, Stewart et al. (2012), à la suite de Duvendack et al. (2011), concluent que les études disponibles «ne trouvent pas un lien de causalité solide entre l'augmentation de l'accès à la microfinance et la réduction de la pauvreté ou le bien-être social pour les pauvres ». Les méthodes d'évaluation par assignation aléatoire, qui ont récemment renouvelé les méthodologies d'évaluation, ne semblent pas plus à même d'établir la causalité : pour le J-PAL (Abdul Latif Jameel Poverty Action Lab) et l'IPA (Innovations for Poverty Action) (2015), sur la base de sept évaluations randomisées, il apparaît que "le microcrédit n'a pas un impact transformateur sur la pauvreté " et que "l'accès au microcrédit n'a pas conduit à une augmentation substantielle des revenus». 
L'apparent succès du microcrédit pendant sa première période de développement était en partie dû à une mesure erronée de sa réussite, basée principalement sur le taux de remboursement. Mais celui-ci est réducteur dès lors que l'on s'intéresse aux diverses stratégies mises en œuvre par les emprunteurs pour permettre ce remboursement. S'il peut être le fruit de la création ou du développement d'une activité génératrice de revenus, comme le prévoit le modèle standard du microcrédit, il peut aussi être expliqué par de multiples autres raisons dont certaines en contradiction avec les objectifs initiaux du microcrédit (Servet, 2015).

Le microcrédit ne se substituant pas aux pratiques financières informelles (Roesch et Helies, 2010), il peut en outre exposer les individus à un risque supérieur de surendettement (Guérin et al., 2014). Cela explique en grande partie les diverses crises d'impayés dont ont été victimes les institutions de microfinance en plusieurs régions du monde (Guérin et al., 2015). Pour certains de ses détracteurs, le microcrédit ne serait même pas souhaitable du point de vue du développement économique car il perpétue une économie de l'informalité, faite d'autoemplois, de microentreprises et de petits commerces (Bateman, 2010). D’une manière générale, enfin, la microfinance bénéficie souvent à une minorité relativement privilégiée plutôt qu'aux plus pauvres (Servet, 2006 et 2015 ; Guérin, 2015).

Les réussites du microcrédit apparaissent en définitive comme hautement contingentes à la situation locale dans laquelle il est mis en œuvre. La réalisation de la chaîne causale " microprêt + opportunité économique $=$ investissement productif + augmentation des revenus + élévation du niveau de vie " dépend donc d'un certain nombre de conditions qu'il s'agit de ne pas ignorer. Servet (2015) identifie ainsi onze conditions nécessaires, qui doivent être réunies aux niveaux microéconomique, mésoéconomique et macroéconomique pour que le microcrédit participe effectivement à la réduction de la pauvreté. Parmi ces conditions, on peut notamment citer l'existence de débouchés pour la production qui est financée, le fait que les gains des uns ne se fassent pas au détriment des autres ou que les flux financiers n'entraînent pas un drainage des ressources locales.

\section{LES BANQUES COMMUNAUTAIRES DE DÉVELOPPEMENT ET LEURS MONNAIES SOCIALES}

Les banques communautaires de développement constituent un exemple d'organisations de microfinance autogérées présentant une alternative solidaire aux pratiques du secteur. Elles mettent en œuvre une stratégie intégrée de développement local, notamment organisée 
autour de l'émission de différents types de microcrédits. Certains sont émis en monnaie nationale, pendant que d'autres sont émis en monnaie dite "sociale" ou "locale". Ces monnaies, propres à chacune des banques communautaires de développement, ne circulent que sur le territoire servi par la banque émettrice et ne sont acceptées et utilisées que sur une base volontaire. Il n'y a pas de convertibilité possible entre elles. Elles partagent néanmoins les mêmes modalités, en étant à parité avec la monnaie nationale et émises notamment à travers des microcrédits à la consommation. Il existe aujourd'hui cent dix banques communautaires au Brésil, présentes sur presque tout le territoire brésilien, et principalement dans la région du Nordeste du fait de la présence de la Banque Palmas qui a joué un rôle pionnier avant de diffuser sa méthodologie. Ainsi nous revenons d'abord sur son histoire, pour mieux comprendre comment ses méthodes ont ensuite été répliquées.

\section{Une "banque " ancrée dans son territoire}

La Banque Palmas représente un cas singulier d'organisation de microfinance gérée par une communauté : son modèle organisationnel est très différent de celui développé par les institutions de microfinance traditionnelles. Elle n'a d'ailleurs de banque que le nom puisqu'elle n'a pas le statut bancaire, mais est une structure non gouvernementale sans but lucratif. Elle a émergé au sein du Conjunto Palmeiras, une zone pauvre et périphérique de la grande ville de Fortaleza, située dans le Nordeste brésilien. Le quartier a surgi en 1973 d'une zone marécageuse couverte d'une végétation dense et dépourvue de toute infrastructure, lorsque y ont été regroupées des populations expulsées de zones urbaines à risque ou du littoral pour cause de spéculation immobilière (Melo et al., 2009). En grande partie livrés à eux-mêmes, les habitants se sont progressivement organisés via certaines modalités d'action collective et d'organisation populaire. Cette mobilisation s'organisa autour de l'Association des habitants du Conjunto Palmeiras (Associação de Moradores do Conjunto Palmeiras, communément appelée ASMOCONP), créée en 1981 et dont les conférences populaires "Habiter l'inhabitable " avaient alors pour but de planifier le développement sur le long terme, par la détermination d'objectifs prioritaires et la définition des modalités d'action à mettre en œuvre.

Les actions menées permirent un aménagement progressif du quartier et une amélioration graduelle des conditions de vie. La pauvreté restait néanmoins globalement importante parmi les habitants du quartier. De plus, l'urbanisation et l'augmentation relative du niveau de vie liées aux mobilisations sociales engendrèrent de nouveaux coûts que les 
premiers habitants ne pouvaient assumer (Melo, 2011). Émergea alors comme une nécessité le fait d'agir sur l'économie locale elle-même. Le manque d'accès au crédit et les difficultés de commercialisation représentaient un frein majeur pour les petits producteurs et les entrepreneurs locaux. Partant de ces constats, l'ASMOCONP entama un processus consultatif, regroupant habitants, commerçants, producteurs et leaders du quartier, pour savoir comment renforcer l'économie locale et faciliter la circulation des revenus au sein du quartier. C'est à la suite de ces débats qu'est née la Banque Palmas en janvier 1998.

\section{Redéfinir l'usage de la monnaie}

Sa fondation repose sur des constats simples, mais néanmoins importants à propos des dynamiques qui étaient à l'œuvre sur le territoire. Face à l'interrogation quant aux raisons de la persistance de la pauvreté, la réponse courante était : « Nous sommes pauvres car nous n'avons pas d'argent. " Mais ce constat fut remis en cause par une enquête de l'ASMOCONP réalisée en 1997 et qui révéla que $80 \%$ de la consommation des ménages était réalisée à l'extérieur du quartier (Melo et al., 2009). Ainsi la communauté n'était pas intrinsèquement pauvre, mais s'appauvrissait en raison de la fuite des revenus vers le centre-ville. La réponse apportée par l'association et ses leaders communautaires était donc: "Nous sommes pauvres car nous perdons notre argent. »

Pour sortir de cette situation, il fallait procéder à une relocalisation des capitaux et, plus généralement, générer une croissance économique locale, sans pour autant se cantonner à des indicateurs purement économiques. La logique de développement territorial intégré conçue par la Banque Palmas s'inscrit dans une création de richesse multidimensionnelle autant quantitative (création d'emplois, augmentation des revenus, etc.) que qualitative (génération de cohésion sociale, lutte contre la violence conjugale, accès à l'éducation, etc.). Ainsi la Banque Palmas vise à renforcer un développement conçu comme un renforcement des forces endogènes du quartier (Meyer, 2015). Il s'agit de promouvoir les capacités territoriales à travers la création et la dynamisation de réseaux de producteurs et de consommateurs locaux.

Pour ce faire, la Banque Palmas propose notamment deux lignes de crédit : l'une en monnaie nationale pour les crédits à la production, l'autre en monnaie locale, nommée "Palmas", pour les crédits à la consommation. Cette articulation d'instruments de crédit en deux monnaies vise, d'une part, à soutenir l'offre locale de biens et de services et, d'autre part, à renforcer la demande interne à travers la concentration du pouvoir d'achat vers les entreprises de ces communautés. 


\section{L'association entre monnaie sociale et microcrédit}

Créée en 2002, la monnaie Palmas est l'une des multiples formes possibles de monnaies dites "sociales" (Blanc, 2006). Elle est une monnaie manuelle qui prend la forme de billets. À parité avec la monnaie nationale, elle est émise par la banque communautaire, qui en assure la gestion. Il n'y a pas de création monétaire lors de son émission : chaque unité de monnaie locale est couverte par une unité de monnaie nationale gardée en réserve par la banque communautaire. La monnaie Palmas vise le renforcement de l'économie locale en incitant ses utilisateurs à réaliser leurs achats au sein du quartier, favorisant de facto la localisation des échanges et leur dynamisation (Fare et al., 2015).

Le crédit à la consommation est destiné à aider les familles quand elles se trouvent dans des situations d'urgence et ne sont pas en mesure d'y subvenir financièrement. C'est lorsque le microcrédit permet de lisser les flux de trésorerie des ménages et de faire face aux chocs qu'il apparaît le plus utile : par exemple, quand le gaz de cuisine se termine ou quand il faut se procurer des médicaments en cas de maladie. Limité à 50 Palmas (15 euros) pour le premier crédit et pouvant atteindre 300 Palmas (90 euros) si la personne rembourse à temps, ce type de crédit est gratuit, aucun taux d'intérêt n'est appliqué : c'est un type de crédit solidaire. Seule une taxe administrative de $1 \%$ est appliquée pour couvrir en partie les frais de gestion. Le prêt est délivré uniquement en monnaie Palmas et ne peut donc être dépensé qu'à l'intérieur du quartier. Ce système permet à la consommation ainsi financée de bénéficier prioritairement à l'économie locale, à la demande supplémentaire d'être adressée aux entrepreneurs du quartier. L'effet multiplicateur est ainsi maximisé. Les microcrédits à la production sont quant à eux émis en réaux brésiliens. Le fait qu'ils soient émis dans la monnaie nationale permet aux entrepreneurs d'acquérir en dehors de la communauté les moyens de production dont ils ont besoin pour développer leurs activités au sein de la communauté. Ces crédits ont un montant de 50 à 15000 réaux brésiliens ( 15 euros à 4500 euros) et sont émis à un taux d'intérêt mensuel de $1,5 \%$ à $3,5 \%$. Les deux types de microcrédits permettent donc d'ancrer localement offre et demande, et de créer un réseau articulé de producteurs et de consommateurs. Cette méthodologie a permis une internalisation progressive de la consommation au sein du Conjunto Palmeiras. En 2008, 95 \% des achats des habitants de la communauté étaient réalisés dans le quartier, contre $20 \%$ en 1997.

Les modalités de décision relatives au crédit font également la spécificité des banques communautaires. Outre les critères classiques, la 
décision des agents de crédit se base aussi sur la mobilisation des réseaux d'interconnaissance au sein de la communauté. Tout le monde peut ainsi avoir accès au crédit, indépendamment des garanties matérielles qu'il peut apporter, même les personnes inscrites au registre national des interdits bancaires (ces personnes devant tout de même présenter un garant). Malgré cela, le taux de non-remboursement n'est que de $2,5 \%$, inférieur aux $6 \%$ à $8 \%$ des banques commerciales (França $e t$ al., 2012).

La monnaie sociale trouve ici sa place au sein d'une méthodologie intégrée de développement dont l'objectif est la restructuration de l'économie locale. La fourniture de microcrédits est en effet couplée avec d'autres types de services et d'interventions. La banque communautaire fournit d'abord des services de correspondance bancaire en partenariat avec les banques nationales, ce qui favorise l'inclusion financière formelle. Mais elle participe également à une construction active de l'économie locale, notamment à travers des cartographies de la production et de la consommation. Ces cartographies répertorient les différents biens et services produits par les acteurs formels et informels du quartier, incluant des données telles que l'âge de l'entreprise, les lieux de vente, le chiffre d'affaires, les accès et les besoins en services financiers, ainsi que les actions personnelles au sein de la communauté (participation dans une organisation sociale, par exemple). Elles recensent aussi les niveaux de scolarisation, de revenus, les types d'emplois (formels ou informels) ainsi que les volumes et les habitudes de consommation des habitants. Elles sont utilisées par la banque pour mieux connaître son territoire d'implantation et ainsi déterminer quelles actions mener.

Pendant longtemps, les orientations de la banque étaient décidées au sein d'un forum local ouvert, réunissant différents acteurs du quartier décidés à débattre des questions liées à la vie en communauté. Ce forum se réunit encore occasionnellement pour mobiliser la population sur des sujets d'intérêt commun. Par exemple, à la fin de 2016, la Banque Palmas a organisé une grande réunion pour débattre et échanger sur le cadastre et la régularisation des habitations dans le quartier. Elle met un point d'honneur à conserver cette dynamique de débat public sur les questions d'intérêt communautaire. Cette volonté se retrouve également dans sa gouvernance. Son conseil d'administration associe en effet plusieurs leaders engagés dans des actions sociales ou des activités culturelles, ainsi que les bénéficiaires des projets de la banque. L'implication de ces personnes dans la gouvernance vise à garantir un contrôle social de la banque et l'articulation de ses activités avec les besoins de la communauté. 


\section{LA COCONSTRUCTION D'UNE POLITIQUE DE DÉVELOPPEMENT ENDOGÈNE}

\section{La diffusion de la méthodologie}

La méthodologie Palmas fut progressivement sollicitée par d'autres communautés en situation de précarité. Sa dissémination se réalisa d'abord principalement dans l'Etat du Ceara, d'où la Banque Palmas est originaire. Au début des années 2000, ses dirigeants organisèrent une "caravane" voyageant dans différentes municipalités de l'État pour en faire connaître la méthodologie. À cette connaissance locale vient s'ajouter l'intérêt du mouvement de l'économie solidaire qui est en plein essor au Brésil à cette époque. Ainsi plusieurs banques communautaires ont émergé dans l'État du Ceara tout d'abord, mais aussi dans l'ensemble du Brésil grâce à l'action d'organisations intermédiaires comme des incubateurs universitaires (Virolle et al., 2016) ou des ONG répliquant la méthodologie. La diffusion s'accéléra notamment grâce à des partenariats avec le secrétariat national de l'économie solidaire, qui a soutenu la création de banques communautaires au Ceara dès 2005, puis au niveau national dès 2010. Ainsi, de trois en 2005, les banques communautaires étaient au nombre de trente-sept en 2008, puis soixante-dix-huit en 2012, pour atteindre cent dix aujourd'hui.

Cette diffusion incita la Banque Palmas à se doter d'une autre structure institutionnelle lui permettant à la fois de diffuser son expérience et de répondre à des appels publics : l'Institut Palmas. Depuis 2003, l'Institut Palmas diffuse l'expérience acquise et, depuis 2004, accompagne la construction d'autres banques communautaires. Cette réplication de l'expérience fut activement soutenue par plusieurs agences de promotion de l'économie solidaire aux niveaux local, étatique et national. Des espaces de négociation ont été mis en place dans les gouvernements à ces différents échelons, ce qui permet aux banques communautaires d'interagir avec les autorités et les banques publiques. Ainsi les banques communautaires de développement participent à la coconstruction de plusieurs politiques locales d'inclusion financière dans un objectif de réduction de la pauvreté.

Toutefois, n'ayant pas le statut bancaire, les banques communautaires ne peuvent capter l'épargne et doivent établir des partenariats avec des banques traditionnelles pour augmenter leur portefeuille et diversifier leur offre de services financiers. Cela passe avant tout par des partenariats avec les banques publiques nationales (Melo et Braz, 2013 ; Meyer, 2015). La Banque Palmas emprunta d'abord à la Banco Popular do Brasil et à la BNDES (Banco Nacional de Desenvolvimento Economico e Social), la banque nationale de développement, pour augmenter 
son capital et développer son portefeuille de crédits. Ces partenariats furent respectivement facilités par le développement du Programme national de microcrédit productif orienté, organisé par le premier gouvernement Lula, puis par le développement d'un département d'économie solidaire à la BNDES. La Banque Palmas est également le correspondant bancaire de la banque Caixa Econômica Federal chargée des transferts de la majorité des programmes sociaux fédéraux, dont la Bolsa Família. Ces activités permettent à la Banque Palmas d'offrir un large éventail de services, dont la possibilité de réaliser des paiements bancaires ou de réceptionner les transferts sociaux.

Ces partenariats sont de toute évidence facilités par la reconnaissance des droits d'auto-organisation des communautés liés à la gestion d'une banque communautaire. Or cette reconnaissance fut difficile, puisque la Banque Palmas a suscité l'attention - et la suspicion - de la Banque centrale du Brésil (BCB) dès ses origines en 1998 (Melo et al., 2009). À l'époque, elle voulait capter l'épargne des habitants. Or, comme elle n'était pas officiellement une institution financière, elle ne pouvait pas légalement proposer de services bancaires. Aussi la BCB lui ordonna de cesser ses activités relatives à l'épargne. Par la suite, en 2003, le procureur de la République l'a assignée en justice en raison de la monnaie Palmas. Le motif invoqué était que l'émission d'une monnaie fiduciaire allait contre les compétences constitutionnelles exclusives de la $\mathrm{BCB}$ en termes d'émission monétaire et de régulation de l'offre de monnaie. Après une enquête de terrain, la BCB émit un avis favorable à la monnaie sociale, reconnaissant qu'elle était développée par des populations traditionnellement marginalisées du système financier et monétaire. Elle remplissait donc des objectifs sociaux et de développement que la monnaie nationale ne réussissait pas à remplir. Se basant sur ce rapport, le procureur de la République en charge du dossier reconnut la mission sociale de la monnaie complémentaire et arrêta les poursuites. Il existe aujourd'hui une note technique cosignée par la $\mathrm{BCB}$ et le ministère du Travail reconnaissant la légalité des monnaies sociales de type Palmas.

L'inexistence d'un statut juridique adapté aux banques communautaires représente néanmoins un risque pour la continuité de telles expériences. Tout d'abord, si l'utilisation de monnaies sociales a été tolérée et soutenue par certaines institutions fédérales, le changement de gouvernement en 2016 ne devrait pas encourager leur développement. En effet, l'une des premières actions du gouvernement Temer a été de supprimer le secrétariat national à l'économie solidaire, qui soutenait les banques communautaires dans leur diffusion et leur consolidation. L'obligation qui leur est faite d'emprunter des capitaux auprès des grandes banques brésiliennes a également un coût pour ces structures. Dans le but d'élargir leurs possibilités d'action, et notam- 
ment de collecter l'épargne, et bénéficiant de certains appuis politiques, les banques communautaires ont fait porter une loi relative à la finance solidaire au Parlement fédéral. Cette loi n’a cependant jamais été adoptée.

\section{La naissance d'une monnaie sociale digitale}

Comme on l'a vu, la monnaie Palmas a entraîné une longue réflexion sur le rôle de la monnaie et de la finance dans les communautés périphériques. Elle a eu un impact symbolique sur la communauté du Conjunto Palmeiras en affirmant une identité positive du territoire (Fare et al., 2015) et a doté la Banque Palmas d'une grande notoriété internationale parmi les acteurs des monnaies sociales. Toutefois sa monnaie a subi de nombreux écueils et son usage s'est réduit. En effet, des études de terrain successives, menées en 2010-2011, puis en 20142015 par l'un des auteurs, montrent une diminution drastique de l'usage de la monnaie sociale. Si la banque communautaire avait émis plus de 80000 Palmas au cours de l'année 2011, ce montant se limitait à quelques milliers en 2014. L'une des causes est un changement de législation qui n'autorise plus les employés de la banque à percevoir une partie de leur salaire en monnaie Palmas, alors que cela constituait une modalité importante d'émission. Une autre hypothèse est que la monnaie sociale a surtout un rôle éducatif et transitoire : à la suite de la relocalisation de la production et de la consommation, la monnaie aurait rempli sa mission et son emploi direct ne serait plus nécessaire. Certaines des nouvelles actions de la banque, telles que le développement du "Palminhas " en direction des enfants, se concentrent ainsi sur le volet symbolique de la monnaie.

Néanmoins, pour capitaliser sur le travail accompli et offrir une forme de monnaie sociale plus adaptée aux pratiques actuelles, la Banque Palmas en a développé une version électronique, qui pourrait redynamiser la circulation en palliant les limites de la monnaie papier (Diniz et al., 2016). Dénommée " e-dinheiro ", cette monnaie s'échange via les téléphones portables. Nouvellement lancée en mai 2016, elle comptait 2235 usagers en septembre 2016, pour une valeur échangée de plus de 2 millions de réaux durant le mois (environ 650000 euros). Le e-dinheiro circule grâce à une application mobile unique, utilisable par les clients de toutes les banques communautaires. L'utilisateur se voit néanmoins fournir un compte par sa banque communautaire locale, à laquelle son compte en e-dinheiro est lié. Le real est ainsi en partie circonscrit au local. Ce système doit offrir des services de paiement et de transfert plus avantageux que les alternatives commerciales et les revenus tirés des services fournis doivent servir à l'investissement dans les communautés respectives des banques communautaires de développement. 


\section{CONCLUSION}

On l'a vu, la microfinance, dans sa mise en œuvre à travers le modèle classique du microcrédit, montre des limites. Celles-ci peuvent notamment être d'ordre économique, relatives aux caractéristiques des territoires, ou d'ordre social, relatives à l'appropriation des dispositifs par leurs bénéficiaires et la perception qu'ils en ont. Les banques communautaires de développement brésiliennes, à travers leur méthodologie inclusive, introduisent des innovations venant en partie répondre à ces limites et renouvellent de façon tout à fait intéressante les modalités du microcrédit, en y associant notamment l'émission d'une monnaie complémentaire.

Mais si la monnaie sociale joue ici un rôle économique, elle dépasse cette seule dimension. On remarque qu'elle est aussi un outil de mobilisation, d'éducation et de sensibilisation, autant qu'un outil symbolique (Fare et al., 2015). Outre ses impacts directs, elle permet également aux communautés à faibles revenus de mieux comprendre les aspects monétaires de l'économie (Braz et al., 2014) à travers la participation du plus grand nombre à la construction de cet outil. Par ailleurs, elle agit sur les comportements en sensibilisant à l'importance d'une consommation locale dans une perspective de développement territorial.

Les banques communautaires, en mobilisant des ressources financières au profit des territoires pauvres, en transforment également la nature (Hudon et Meyer, 2016). À travers l'action d'institutions qui sont le fruit de l'auto-organisation des membres de la communauté, par les dynamiques de gouvernance collective et grâce à l'appropriation des outils mis en place, la ressource financière mobilisée devient une ressource commune, au service du territoire, ici entendue dans sa spatialité, mais aussi dans sa socialité constitutive. Alors que dans le cas du microcrédit classique, «l'origine externe des fonds les apparente à de l'argent froid " et que cette absence de lien avec la communauté locale fait que l'obligation de rembourser est pensée comme faible par les populations (Servet, 2006), les banques communautaires et leurs bénéficiaires partagent un même projet et des intérêts communs. La tension interne à la microfinance classique entre recherche d'autonomie financière et réalisation de l'impact social est relâchée par le modèle organisationnel des banques communautaires, et notamment sa gouvernance collégiale. De nombreuses organisations de microfinance ont fait l'objet de dérives, précisément car leur gestion ne prenait pas assez en compte l'intérêt des bénéficiaires.

La méthodologie des banques communautaires de développement s'inscrit donc dans une démarche de finance solidaire, dont les pratiques « visent à instrumentaliser le crédit » au service d'objectifs socio- 
économiques, mais dans laquelle " créanciers et débiteurs partagent une fin commune " de développement collectif. Dans cette approche, les pratiques "inscrivent les relations débiteurs-créanciers dans un cadre collectif dépassant les limites du simple échange contractuel " (Ferraton, 2006). Plus largement, les banques communautaires participent à la mise en place d'une économie solidaire "non au sens d'expériences sympathiques et conviviales animées par quelques militants désireux de réhumaniser l'économie - éternel cliché qui masque les véritables enjeux de l'économie solidaire -, mais au sens d'une véritable démocratisation des pratiques économiques" (Guérin, 2004).

La méthodologie des banques communautaires de développement offre finalement un contre-point tout à fait intéressant au paradigme d'inclusion financière universelle tel qu'actuellement promu et mis en œuvre (Doligez et al., 2016). À l'idée que chaque individu est voué à être naturellement entrepreneur et doit s'insérer de façon autonome à l'économie de marché grâce à la mobilisation des outils financiers qui lui sont offerts est opposée une logique de construction et de mobilisation collective en faveur d'un développement endogène et collectif. L'action des banques communautaires montre qu'une approche par le seul microcrédit classique est restrictive puisqu'elle présume que le seul problème des populations pauvres est la difficulté d'accès au capital. Mais un processus de développement ne saurait se réduire à ce seul paramètre.

Ainsi, plutôt que d'individualiser les situations de chacun en oubliant le caractère systémique du changement, sont favorisés des processus collectifs qui s'organisent autour de projets monétaires à même de cristalliser des dynamiques de développement endogène. Dans le cas de la Banque Palmas, "au-delà d'une institution traditionnelle de microfinance, cette banque communautaire vise le développement du quartier comme un tout et non comme celui d'individus isolés. Elle part du principe que personne ne vainc la pauvreté seul » (Melo, 2011).

\section{NOTE}




\section{BIBLIOGRAPHIE}

Bateman M. (2010), Why Doesn't Microfinance Work? The Destructive Rise of Local Neoliberalism, Zed Books, 274 p.

BCB (Banque centrale du Brésil) (2015), Relatório de Inclusão Financeira, n 3, www.bcb.gov.br/ Nor/relincfin/RIF2015.pdf.

BLANC J. (2006), « Les monnaies sociales : un outil et ses limites. Introduction générale », in Blanc J. (éd.), Exclusion et liens financiers : monnaies sociales, rapport 2005-2006, Economica, pp. 11-23.

Braz J., Neiva A. C. et Nakagawa C. T. (2014), "Community Development and Social Currency: Main Results of the Banco Palmas Experience", Veblen Institute for Economic Reforms, www.veblen-institute.org/Community-development-and-social.

Diniz E. H., Cernev A. K. et Nascimento E. (2016), « Mobile Social Money: an Exploratory Study of the Views of Managers of Community Banks ", Revista de Administração, vol. 51, $\mathrm{n}^{\circ} 3$.

Doligez F., Bastiaensen J., BédéCARrats F. et Labie M. (2016), « L'inclusion financière, nouvel avatar de la libéralisation financière ? ", Revue Tiers Monde, n² 225, 5 avril, pp. 9-20.

Duvendack M., Palmer-Jones R., Copestake J. G., Hooper L., Loke Y. et Rao N. (2011), "What Is the Evidence of the Impact of Microfinance on the Well-Being of Poor People? ", EPPI-Centre, Social Science Research Unit, Institute of Education, University of London.

Fare M., De Freitas C. et Meyer C. (2015), «Territorial Development and Community Currencies: Symbolic Meanings in Brazilian Community Development Banks ", International Journal of Community Currency Research, vol. 19, https:/ijccr.net/2015/02/25/territorial-development/.

Ferraton C. (2006), "Finance solidaire ", in Laville J.-L. et Cattani A. D. (éd.), Dictionnaire de l'autre économie, édition revue et augmentée, Folio.

Fouillet C., Guérin I., Morvant-Roux S., Roesch M. et Servet J.-M. (2007), « Le microcrédit au péril du néolibéralisme et de marchands d'illusions. Manifeste pour une inclusion financière socialement responsable ", Revue du MAUSS, vol. 29, n 1, pp. 329-350, www.cairn.info/revue-du-mauss-20071-page-329.htm.

França F. G., Scalfoni R. A., Torres S. J. J. et Meyer C. (2012), «L'enjeu de l'usage des monnaies sociales dans les banques communautaires de développement au Brésil : étude du cas de la Banque Palmas ", Revue internationale de l'économie sociale, n 324, pp. 70-86.

França F. G. et Torres S. J. J. (2009), "Bancos comunitarios de desenvolvimento ", in Cattani A., Laville J.-L., Gaiger L. et Hespanha P. (dir.), Dicionario internacional da outra economia, Ediçóes Almedina Brasil, pp. 31-36.

GuÉRIN I. (2004), « Conclusion : la microfinance, limites et espoirs ", in Guérin I. et SERVET J.-M. (éd.), Exclusion et liens financiers : microfinance, leçons du sud, rapport du Centre Walras 2003, Economica.

GUÉRIN I. (2015), La microfinance et ses dérives : émanciper, discipliner ou exploiter ?, Demopolis.

Guérin I., Labie M. et Servet J.-M. (éd.) (2015), The crises of microcredit, Zed Books.

Guérin I., Morvant-Roux S. et Villarreal M. (éd.) (2014), Microfinance, Debt and Over-Indebtedness: Juggling with Money, Routledge, coll. " Routledge Studies in Development Economics ", 316 p.

Hudon M. et MeYer C. (2016), «A Case Study of Microfinance and Community Development Banks in Brazil Private or Common Goods? ", Nonprofit and Voluntary Sector Quarterly.

J-PAL et IPA(2015), "Where Credit Is Due ", JPAL/IPA Policy Bulletin, www.povertyactionlab.org/sites/default/files/publications/where-credit-is-due.pdf.

Melo J. (2011), "Banco Palmas ou les richesses d'une favela ", Revue Projet, vol. 324-325, n 5 , $1^{\text {er }}$ décembre, pp. 114-117.

Melo J., Becu E. et de Freitas C. (2009), Viva Favela! Quand les démunis prennent leur destin en main, Michel Lafon, 281 p.

Melo J. et Braz J. (éd.) (2013), Banco Palmas - Resistindo e Inovando, A9 Editora.

Meyer C. (2015), «La pluralité des logiques d'action de la Banque Palmas au Brésil : entre développement local et partenariats au niveau national ", Revue française de socio-économie, vol. 15, $\mathrm{n}^{\circ} 1,23$ avril, pp. 59-76. 
Roesch M. et Helies O. (2010), « La microfinance, outil de gestion du risque ou de mise en danger par surendettement ?", Autrepart, n 44, 1' ${ }^{\text {er }}$ mars, pp. 119-140.

Schiavinatto F. et De Holanda Schmidt F. (2011), «SIPS Bancos. Inclusão financeira e bancarização no Brasil ", in Schiavinatto F. (org.), Sistema de indicadores de percepção social, IPEA, pp. 181-210.

SERVET J.-M. (2006), Banquiers aux pieds nus : la microfinance, Odile Jacob, 511 p.

SERVET J.-M. (2015), La vraie révolution du microcrédit, Odile Jacob, 256 p.

Soares M. M. et De Melo Sobrinho A. D. (2008), Microfinanças: o papel do Banco Central do Brasil e a importancia do Cooperativismo de Crédito, Banque centrale du Brésil, www.bcb.gov.br/htms/public/microcredito/livro_microfinan\%E7as_2007_internet.pdf.

Stewart R., van Rooyen C., Korth M., Chereni A., Rebelo da Silva N. et De Wet T. (2012), Do Micro-Credit, Micro-Savings and Micro-Leasing Serve as Effective Financial Inclusion Interventions Enabling Poor People, and Especially Women, to Engage in Meaningful Economic Opportunities in Low- and Middle-Income Countries? A Systematic Review of the Evidence, EPPI-Centre, Social Science Research Unit, Institute of Education, University of London.

Virolle L., Pozzebon M. et Gonzalez L. (2016), «Les incubateurs technologiques de coopératives : L'expérience des universités brésiliennes ", Revue internationale de l'économie sociale, nº 340, p. 110-125. 